\title{
Nonexistence of Nontrivial Stationary Solutions with Decay Order for Some Nonlinear Evolution Equations
}

\author{
Jeng-Eng $\operatorname{Lin}^{1}$ \\ ${ }^{1}$ Department of Mathematics and Statistics, Georgetown University, Washington, DC, USA \\ Correspondence: Jeng-Eng Lin, Department of Mathematics and Statistics, Georgetown University, Washington, DC \\ 20057, USA. E-mail: JengEng.Lin@georgetown.edu
}

Received: March 21, 2017 Accepted: April 8, 2017 Online Published: May 3, 2017

doi:10.5539/jmr.v9n3p1

URL: https://doi.org/10.5539/jmr.v9n3p1

\section{Abstract}

We show that there are no nontrivial stationary solutions of certain decay order for some applied nonlinear evolution equations which include the thin epitaxial film model with slope selection and the square phase field crystal (SPFC) equation. The method is to use the Morawetz multiplier and the Gagliardo-Nirenberg inequality.

\section{Mathematics Subject Classifications Primary 35J60, 35J61, 35J62.}

Keywords: thin epitaxial film model, square phase field crystal equation, Gagliardo-Nirenberg inequality, Morawetz multiplier.

\section{Introduction}

Consider the thin epitaxial film model with slope selection (Li \& Liu, 2004; Shen, Wang, Wang, \& Wise, 2012; Wang, Wang, \& Wise, 2010; Xu \& Tang 2006),

$$
\frac{\partial \mathrm{u}}{\partial \mathrm{t}}=-\varepsilon^{2} \Delta^{2} u+\nabla \cdot\left(|\nabla u|^{2} \nabla u\right)-\Delta u
$$

and the square phase field crystal equation (Cross \& Hohenberg, 1993; Hernández, Castañeda, \& Cadenas, 2014; Hoyle, 1995; Lloyd, Sandstede, Avitabile, \& Champneys, 2008),

$$
\frac{\partial \mathrm{u}}{\partial \mathrm{t}}=\Delta\left(\gamma_{0} u+\gamma_{1} \Delta u+\varepsilon^{2} \Delta^{2} u-\nabla \cdot\left(|\nabla u|^{2} \nabla u\right)\right)
$$

The stationary equation of (1.1) is

$$
-\varepsilon^{2} \Delta^{2} u+\nabla \cdot\left(|\nabla u|^{2} \nabla u\right)-\Delta u=0
$$

that is,

$$
\varepsilon^{2} \Delta^{2} u-\nabla \cdot\left(|\nabla u|^{2} \nabla u\right)+\Delta u=0
$$

The stationary equation of (1.2) is

$$
\Delta\left(\gamma_{0} u+\gamma_{1} \Delta u+\varepsilon^{2} \Delta^{2} u-\nabla \cdot\left(|\nabla u|^{2} \nabla u\right)\right)=0 .
$$

Thus

$$
\gamma_{0} u+\gamma_{1} \Delta u+\varepsilon^{2} \Delta^{2} u-\nabla \cdot\left(|\nabla u|^{2} \nabla u\right)=0
$$

if the solution decays at infinity.

In this paper, we will study the equation that is more general than the above equations (1.3) and (1.4), 


$$
\Delta^{2} u-\nabla \cdot\left(|\nabla u|^{p-2} \nabla u\right)+a \Delta u+b u+f(x, u)=0,
$$

where $\mathrm{a}$ and $\mathrm{b}$ are constants and $\mathrm{f}$ is a continuous function. We will show that under certain conditions of $\mathrm{a}, \mathrm{b}$, and $f(x, u)$, there is no nontrivial solution with certain decay order for (1.5). The method is to use the Morawetz multiplier (Levandosky \& Strauss, 2000; Morawetz 1968) and the Gagliardo-Nirenberg inequality (Bellazzini, Frank, \& Visciglia, 2014).

Nonlinear fourth-order elliptic equations related to (1.5) have attracted a lot of attentions lately. For example, for the equation $-\Delta^{2} u=u^{p}$, it is known (Lin 1998; Wei and Xu 1999; Fazly, Wei, \& Xu 2015) that it doesn't have a nontrivial nonnegative solution when $1<p<\frac{n+4}{n-4}$ with $n \geq 5$, and $p>11$ with $n \leq 4$. Also for the case of $p=\frac{n+4}{n-4}$, all solutions are classified. Thus it is natural to consider the case of equation (1.5) which combines the effect of nonlinearity, fourth order, and the p-Laplacian operator (Drábek 2007).

As usual, $x=\left(x_{1}, x_{2}, \ldots, x_{n}\right), \nabla u$ denotes the gradient of $\mathrm{u}, \nabla \cdot u$ denotes the divergence of $\mathrm{u}$, and $r=|x|$. Also the subscript denotes the partial derivative, thus $u_{s}=\frac{\partial u}{\partial s}$. We also use the notation $u_{r}=\frac{\partial u}{\partial r}=\frac{x}{r} \cdot \nabla u$ and $\partial_{j}=\frac{\partial}{\partial x_{j}} \cdot F_{r}(x, s)$ denotes $\frac{\partial F(x, s)}{\partial r}=\frac{x}{r} \cdot \nabla_{x} F(x, s) \cdot C^{k}\left(R^{n}\right)$ is the space of functions whose partial derivatives of order up to and including $\mathrm{k}$ are continuously differentiable.

Define the following four sets of functions $\mathrm{D}_{\mathrm{h}, \mathrm{k}}\left(\mathbf{R}^{\mathrm{n}}\right), \mathrm{E}_{\mathrm{m}}\left(\mathbf{R}^{\mathrm{n}}\right), \mathrm{F}_{\mathrm{n}}\left(\mathbf{R}^{\mathrm{n}}\right)$, and $\mathrm{NF}\left(\mathbf{R}^{\mathrm{n}}\right)$, which we will use in this article:

$\mathrm{D}_{\mathrm{h}, \mathrm{k}}\left(\mathbf{R}^{\mathrm{n}}\right)=\left\{u \mid u \in \mathrm{C}^{k}\left(R^{n}\right), \lim _{R \rightarrow \infty}\left(\sup \left(|x| \leq R\left|x^{\alpha}\right|\left|D^{\beta} u(x)\right|\right)\right\}=0\right.$, for all multi-indices $\boldsymbol{\alpha}$ and $\boldsymbol{\beta} \in \mathrm{N}_{0}^{n}$ such that $|\boldsymbol{\alpha}| \leq \mathrm{h}$ and $|\boldsymbol{\beta}| \leq \mathrm{k}$, where $\boldsymbol{\alpha}=\left(\alpha_{1}, \alpha_{2}, \ldots, \alpha_{\mathrm{n}}\right), \boldsymbol{\beta}=\left(\beta_{1}, \beta_{2}, \ldots, \beta_{\mathrm{n}}\right),|\boldsymbol{\alpha}|=\alpha_{1}+\alpha_{2}+\ldots+\alpha_{\mathrm{n}}$, and $\left.|\boldsymbol{\beta}|=\beta_{1}+\beta_{2}+\ldots+\beta_{\mathrm{n}}.\right\}$,

$$
\begin{gathered}
\mathrm{E}_{\mathrm{m}}\left(\mathbf{R}^{\mathrm{n}}\right)=\left\{\mathrm{a} \mid \mathrm{a} \in \mathrm{C}^{1}\left(\mathbf{R}^{\mathrm{n}}\right), \sup _{|x| \geq \rho}\left(|\mathrm{x}|^{-\mathrm{m}}|\mathrm{a}(\mathrm{x})|\right)<\infty \text { for some } \rho>0 \text { and } \mathrm{m}>0\right\}, \\
\mathrm{F}_{\mathrm{n}}\left(\mathbf{R}^{\mathrm{n}}\right)=\left\{\mathrm{u} \mid \lim _{R \rightarrow \infty}\left(\mathrm{R}^{\mathrm{n}} \sup _{|x|=\mathrm{R}}|\mathrm{F}(\mathbf{x}, \mathrm{u}(\mathbf{x}))|\right)=0\right\},
\end{gathered}
$$

And

$$
\mathrm{NF}\left(\mathbf{R}^{\mathrm{n}}\right)=\left\{\mathrm{u} \mid \int_{\mathrm{R}^{n}}\left[\mathrm{nF}(\mathbf{x}, \mathrm{u}(\mathbf{x}))+\mathrm{rF}_{\mathrm{r}}(\mathbf{x}, \mathrm{u}(\mathbf{x}))-((\mathrm{n}-1) / 2) \mathrm{f}(\mathbf{x}, \mathrm{u}(\mathbf{x})) \mathrm{u}(\mathbf{x})\right] \mathrm{d} \mathbf{x} \leq 0\right\},
$$

where $F(\mathbf{x}, u)$ is the antiderivative of $f(\mathbf{x}, u)$ with respect to $u$ such that $F(\mathbf{x}, 0)=0$.

Remark 1. A function $u$ is said to be of decay order $(h, k)$ if and only if $u \in D_{h}, k\left(\mathbf{R}^{n}\right)$. All the functions are assumed to be real-valued.

\section{Morawetz Multiplier}

Multiplying both sides of Equation (1.5) by Morawetz multiplier $\zeta\left(\mathrm{u}_{\mathrm{r}}+((\mathrm{n}-1) \mathrm{u} /(2 \mathrm{r}))\right)$, where $\zeta \in \mathrm{C}^{3}\left(\mathbf{R}^{\mathrm{n}}\right)$ and $\zeta(\mathbf{x})=\zeta(|\mathbf{x}|)$ $=\zeta(\mathrm{r})$, we get

$$
0=\left[\Delta^{2} u-\nabla \cdot\left(|\nabla u|^{p-2} \nabla u\right)+a \Delta u+b u+f(x, u)\right] \zeta\left(u_{r}+\frac{(n-1) u}{2 r}\right)=\nabla \cdot Y+Z,
$$

where $\mathrm{Y}$ depends on $\zeta$ and $\mathrm{u}$ as well as their partial derivatives up to and including the third order and $\mathrm{F}(\mathrm{x}, \mathrm{u})$, and 


$$
\begin{aligned}
& Z=\frac{3 \zeta^{\prime}}{2}(\Delta u)^{2}+A\left(u_{r}\right)^{2}+B\left(|\nabla u|^{2}-\left|u_{r}\right|^{2}\right)+C u^{2}+\left(\zeta-r \zeta^{\prime}\right) P \\
& -\left\{\left(\frac{\zeta}{r}-\zeta\right)|\nabla u|^{p-2}\left[\left(u_{r}\right)^{2}+\frac{n-1}{2 r} u_{r} u\right]+\left[\frac{\zeta^{\prime}}{p}-\left(\frac{\zeta}{2 p r}\right)((n+1) p-2(n-1))\right]|\nabla u|^{p}\right\} \\
& -a\left\{\frac{1}{2} \zeta_{r}|\nabla u|^{2}+(\nabla u \cdot \nabla \zeta) u_{r}-\zeta_{r}\left|u_{r}\right|^{2}+\left(\frac{1}{r} \zeta-\zeta_{r}\right)\left(|\nabla u|^{2}-\left|u_{r}\right|^{2}\right)\right. \\
& \left.+\frac{n-1}{2 r}\left[\frac{1}{r} \zeta_{r}-\frac{1}{2} \Delta \zeta+\frac{n-3}{2 r^{2}} \zeta\right] u^{2}\right\} \\
& +b \frac{\zeta^{\prime}}{2} u^{2}-\left(\zeta_{r}+\frac{n-1}{r} \zeta\right) F(x, u)+\frac{n-1}{2 r} \zeta f(x, u) u-\zeta F_{r}(x, u)
\end{aligned}
$$

where

$$
\begin{aligned}
& A=-\frac{7 \zeta^{\prime \prime}}{2}-\frac{(n-1)(n-3)\left(\zeta^{\prime}-\frac{\zeta}{r}\right)}{2 r^{2}}, \\
& B=\frac{-3 \zeta^{\prime \prime}}{2}+\frac{(n-5) \zeta^{\prime \prime}}{r}-\frac{\left(n^{2}+2 n-19\right)\left(\zeta^{\prime}-\frac{\zeta}{r}\right)}{2 r^{2}}, \\
& C=\frac{n-1}{2}\left[\frac{\zeta^{\prime \prime}}{2 r}+\frac{(n-3) \zeta^{\prime \prime}}{r^{2}}+\frac{(n-3)(n-7) \zeta^{\prime \prime}}{2 r^{3}}-\frac{3(n-3)(n-5)\left(\zeta^{\prime}-\frac{\zeta}{r}\right)}{2 r^{4}}\right],
\end{aligned}
$$

and

$$
P=\frac{2}{r}\left[\sum_{i, j}\left(S_{i, j} u\right)^{2}-\sum_{i}\left(\sum_{j} \frac{x_{j}}{r} S_{i, j} u\right)^{2}\right] \geq 0,
$$

Where $S_{i, j} u=\frac{x_{i}}{r^{3}} \sum_{k}\left[x_{k}\left(x_{k} \partial_{j}-x_{j} \partial_{k}\right) u_{r}\right]+\partial_{j} \sum_{k}\left[\frac{x_{k}}{r^{2}}\left(x_{k} \partial_{i}-x_{i} \partial_{k}\right) u\right]$.

\section{Nonexistence of Nontrivial Solutions}

Theorem Assume $\mathrm{p} \geq(2 \mathrm{n}) /(\mathrm{n}+1)$ and $\mathrm{u} \in \mathrm{D}_{\mathrm{n}, 3}\left(\mathbf{R}^{\mathrm{n}}\right) \cap \mathrm{F}_{\mathrm{n}}\left(\mathbf{R}^{\mathrm{n}}\right) \cap \mathrm{NF}\left(\mathbf{R}^{\mathrm{n}}\right)$.

(a) If $a>0$ and $b>a^{2} C_{n}{ }^{2} / 12$, then $u \equiv 0$, where $C_{n}$ is the constant, depending only on the dimension $n$, for the Gagliardo-Nirenberg inequality.

(b) If $\mathrm{a} \leq 0$ and $\mathrm{b} \geq 0$, then $\mathrm{u} \equiv 0$.

Proof:

Let $R>0$. Integrating both sides of (2.1) in $|\mathbf{x}| \leq R$ and using the Divergence theorem, we get

$$
0=\int_{|x|=R} Y \cdot(x / R) d s+\int_{R^{n}} Z d x
$$

Let $\mathrm{R} \rightarrow \infty$. We get $0=\lim _{R \rightarrow \infty} \int_{|x|=R} Y \cdot(x / R) d s+\int_{R^{n}} Z d x$

Now we let $\zeta(\mathbf{x})=\zeta(|\mathbf{x}|)=\zeta(\mathrm{r})=\mathrm{r}$ in $(2.1)$. Then 


$$
\begin{aligned}
& 0=\int_{R^{n}} Z d x \\
& =\int_{R^{n}}\left\{\frac{3}{2}(\Delta u)^{2}-\frac{2 n-(n+1) p}{2 p}|\nabla u|^{p}-a \frac{1}{2}|\nabla u|^{2}+b \frac{1}{2} u^{2}\right. \\
& \left.-n F(x, u)-\frac{n-1}{2} f(x, u) u-\mathrm{rF}_{r}(x, u)\right\} d x
\end{aligned}
$$

Thus

$$
\begin{aligned}
& \int_{R^{n}}\left\{\frac{3}{2}(\Delta u)^{2}-\frac{2 n-(n+1) p}{2 p}|\nabla u|^{p}-a \frac{1}{2}|\nabla u|^{2}+b \frac{1}{2} u^{2}\right\} d x \\
& =\int_{R^{n}}\left\{n F(x, u)-\frac{n-1}{2} f(x, u) u+\mathrm{rF}_{r}(x, u)\right\} d x
\end{aligned}
$$

To show the assertion (a), we recall the Gagliardo-Nirenberg inequality (Bellazzini, Frank, \& Visciglia, 2014)

$$
\int_{R^{n}}|\nabla u|^{2} d x \leq C_{n}\left(\int_{R^{n}}|u|^{2} d x\right)^{\frac{1}{2}}\left(\int_{R^{n}}|\Delta u|^{2} d x\right)^{\frac{1}{2}}
$$

where $C_{n}$, depending only on $n$, is the least positive constant such that the above inequality (3.2) holds for all $u$ in $H^{2}\left(R^{n}\right)$. Therefore, since a $>0$,

$$
\begin{aligned}
& \frac{a}{2} \int_{R^{n}}|\nabla u|^{2} d x \leq \frac{a C_{n}}{2}\left(\int_{R^{n}}|u|^{2} d x\right)^{\frac{1}{2}}\left(\int_{R^{n}}|\Delta u|^{2} d x\right)^{\frac{1}{2}} \\
& \leq \frac{a^{2} C_{n}^{2}}{24} \int_{R^{2}}|u|^{2} d x+\frac{3}{2} \int_{R^{n}}|\Delta u|^{2} d x
\end{aligned}
$$

Thus

$$
\begin{aligned}
& \int_{R^{n}}\left\{-\frac{2 n-(n+1) p}{2 p}|\nabla u|^{p}+\left[\frac{b}{2}-\frac{a^{2} C_{n}^{2}}{24}\right] u^{2}\right\} d x \\
& \leq \int_{R^{n}}\left\{\frac{3}{2}(\Delta u)^{2}-\frac{2 n-(n+1) p}{2 p}|\nabla u|^{p}-a \frac{1}{2}|\nabla u|^{2}+b \frac{1}{2} u^{2}\right\} d x \\
& =\int_{R^{n}}\left\{n F(x, u)-\frac{n-1}{2} f(x, u) u+r F_{r}(x, u)\right\} d x
\end{aligned}
$$

Since $b>\frac{a^{2} C_{n}^{2}}{12}$ and $p \geq \frac{2 n}{n+1}$.

we have 


$$
\begin{aligned}
& 0 \leq \int_{R^{n}}\left\{-\frac{2 n-(n+1) p}{2 p}|\nabla u|^{p}+\left[\frac{b}{2}-\frac{a^{2} C_{n}^{2}}{24}\right] u^{2}\right\} d x \\
& \leq \int_{R^{n}}\left\{n F(x, u)-\frac{n-1}{2} f(x, u) u+r F_{r}(x, u)\right\} d x \\
& \leq 0,
\end{aligned}
$$

since $\mathrm{u} \in \mathrm{NF}\left(\mathbf{R}^{\mathrm{n}}\right)$.

Thus

$$
\int_{R^{n}}\left\{-\frac{2 n-(n+1) p}{2 p}|\nabla u|^{p}+\left[\frac{b}{2}-\frac{a^{2} C_{n}^{2}}{24}\right] u^{2}\right\} d x=0
$$

Since $u \in \mathrm{D}_{\mathrm{n}, 3}\left(\mathbf{R}^{\mathrm{n}}\right), \mathrm{u} \equiv 0$.

For the assertion (b), since $\mathrm{a} \leq 0, \mathrm{~b} \geq 0$, and $p \geq \frac{2 n}{n+1}$ we have from (3.1),

$$
\begin{aligned}
& 0 \leq \int_{R^{n}}\left\{\frac{3}{2}(\Delta u)^{2}-\frac{2 n-(n+1) p}{2 p}|\nabla u|^{p}-a \frac{1}{2}|\nabla u|^{2}+b \frac{1}{2} u^{2}\right\} d x \\
& =\int_{R^{n}}\left\{n F(x, u)-\frac{n-1}{2} f(x, u) u+r F_{r}(x, u)\right\} d x \\
& \leq 0
\end{aligned}
$$

since $u \in N F\left(\mathbf{R}^{\mathrm{n}}\right)$.

Thus,

$$
\int_{\mathrm{R}^{n}}\left\{\frac{3}{2}(\Delta u)^{2}-\frac{2 n-(n+1) p}{2 p}|\nabla u|^{p}-a \frac{1}{2}|\nabla u|^{2}+b \frac{1}{2} u^{2}\right\} d x=0
$$

Since $\mathrm{u} \in \mathrm{D}_{\mathrm{n}},{ }_{3}\left(\mathbf{R}^{\mathrm{n}}\right), \mathrm{u} \equiv 0$.

Remark 2. As an example for $f(\mathbf{x}, u)$, let $f(\mathbf{x}, u)=q(\mathbf{x})|u|^{s-1} u, s \geq 1$. Then

$$
F(x, u)=\frac{1}{s+1} q(x)|u|^{s+1},
$$

where

$$
q(x)=q(|x|)=q(r)
$$

Assume $\mathrm{q} \in \mathrm{E}_{\mathrm{m}}\left(\mathbf{R}^{\mathrm{n}}\right)$.

For $\mathrm{u}$ to be in $\mathrm{F}_{\mathrm{n}}\left(\mathbf{R}^{\mathrm{n}}\right)$, we need

$$
\lim _{R \rightarrow \infty}\left(R^{n} \sup _{|x|=R}|F(x, u(x))|=0\right.
$$


that is,

$$
\lim _{R \rightarrow \infty}\left(R^{n} \sup _{|x|=R}\left(\mathrm{q}(|x|)|u(x)|^{s+1}\right)\right)=0
$$

This would be satisfied if

$$
\lim _{R \rightarrow \infty}\left(R^{n} \sup _{|x|=R}\left(|x|^{m}|u(x)|^{s+1}\right)\right)=0
$$

The above condition (3.3) would be satisfied if $u$ is of decay order $(n+m, 0)$.

As for $u$ to be in $\mathrm{NF}\left(\mathbf{R}^{\mathrm{n}}\right)$, since

$$
n F(x, u)+r F_{r}(x, u)-\left(\frac{n-1}{2}\right) f(x, u) u=\left[\frac{n}{s+1} q+\frac{r}{s+1} q_{r}-\left(\frac{n-1}{2}\right) q\right]|u|^{s+1}
$$

$\mathrm{u}$ would be in $\mathrm{NF}\left(\mathbf{R}^{\mathrm{n}}\right)$, if $\left(\frac{n}{s+1} q+\frac{r}{s+1} q_{r}-\frac{n-1}{2} q\right) \leq 0$

Thus, if

$$
\mathrm{r} q_{r} \leq\left(\frac{n s-n-s-1}{2}\right) q
$$

then $\mathrm{u}$ is in $\mathrm{NF}\left(\mathbf{R}^{\mathrm{n}}\right)$.

Therefore, if $\mathrm{u}$ is of decay order $(\mathrm{n}+\mathrm{m}, 1)$ and $\mathrm{r} q_{r} \leq\left(\frac{n s-n-s-1}{2}\right) q$, $\mathrm{u}$ satisfies the assumptions of the theorem on $\mathrm{u}$. In particular, $\mathrm{s}$ must $\mathrm{be} \geq(\mathrm{n}+1) /(\mathrm{n}-1)$ if $\mathrm{q}$ is a positive constant and $\mathrm{s}$ must $\mathrm{be} \leq(\mathrm{n}+1) /(\mathrm{n}-1)$ if $\mathrm{q}$ is a negative constant, in order for the inequality (3.4) to hold.

Remark 3. A similar conclusion can be obtained for $\mathrm{f}(x, u)=q_{1}(x)|u|^{\alpha-1} u+q_{2}(x)|u|^{\beta-1} u$, where $\alpha>\beta \geq 1$.

\section{Conclusion}

The classification of entire solutions of nonlinear elliptic equations is one of the intensively investigated topics. Our goal is to completely classify all the solutions of the relatively new equation (1.5). The work in this paper is only a first step in this direction. We will carry out more research in this direction in the future.

\section{Acknowledgement}

A part of this work was carried out while the author was a visiting scholar at the Institute of Mathematics at Academia Sinica, Taipei, during May 19 - June 14 and October 6 - 12, 2016. The author wishes to thank Dr. Jyh-Hao Lee for the hospitality during the visit. The author also wishes to thank the reviewers of this paper for their valuable comments which improve the manuscript.

\section{Reference}

Bellazzini, J., Frank, R. L., \& Visciglia, N. (2014). Maximizers for Gagliardo-Nirenberg inequalities and related non-local problems, Math Ann., 360, 653 - 673 https://doi.org/10.1007/s00208-014-1046-2

Cross, M. C., \& Hohenberg, P. C. (1993). Pattern formation outside of equilibrium, Rev. Mod. Phy., 65, 851 - 1123. https://doi.org/10.1103/RevModPhys.65.851

Drábek, P. (2007). The p-Laplacian - moscot of nonlinear analysis, Acta Math. Univ. Comenianae, LXXVI, Proceedings of Equadiff 11, 85-98

Fazly, M., Wei, J., \& Xu, X. (2015). A pointwise inequality for the fourth-order Lane-Emden equation, Analysis and PDE, 
8, 1541 - 1563. https://doi.org/10.2140/apde.2015.8.1541

Hernández, J. A. M., Castañeda, F. G., \& Cadenas, J. A. M. (2014). Formation of square patterns using a model alike Swift-Hohenberg. $11^{\text {th }}$ international Conference on Electrical Engineering, Computing Science and Automatic Control (CCE), IEEE, 1 - 6. https://doi.org/10.1109/iceee.2014.6978331

Hoyle, R. B. (1995). Steady squares and hexagons on a subcritical ramp, Phys. Rev. E., 51, 310 - 317. Levandosky, S. P., \& Strauss, W. A. (2000). Time decay for the nonlinear beam equations, Methods Appl. Anal., 7, 479 - 488.

Li, B., \& Liu. J. (2004). Epitaxial growth without slope selection: energetics, coarsening, and dynamic scaling. J. Nonlinear Sci., 16, 429 - 451. https://doi.org/10.1007/s00332-004-0634-9

Lin, C.-S. (1998). A classification of solutions of a conformally invariant fourth order equation in $\mathbf{R}^{\mathrm{n}}$, Comment. Math. Helv., 73, 206-231. https://doi.org/10.1007/s000140050052

Lloyd, D. J., Sandstede, B., Avitabile, D., \& Champneys, A. R. (2008). Localized hexagon patterns of the planar Swift-Hohenberg equation. SIAM J. Appl. Dyn. Syst., 7, 1049 -1100. https://doi.org/10.1137/070707622

Morawetz, C. S. (1968). Time decay for nonlinear Klein-Gordon equation, Proc. Roy. Soc. A, 306, 291-296. https://doi.org/10.1098/rspa.1968.0151

Shen, J., Wang, C., Wang, X., \& Wise, S. M. (2012). Second-order convex splitting schemes for gradient flows with Ehrich-Schwoebel type energy: application to the film epitaxy. SIAM J. Numer. Anal., 50, 105 - 125. https://doi.org/10.1137/110822839

Wang, C., Wang, X., \& Wise, S. M. (2010). Unconditionally stable schemes for equations of thin film epitaxy. Discrete Contin. Dyn. Syst., 28, 405 - 423. https://doi.org/10.3934/dcds.2010.28.405

Wei, J., \& Xu, X. (1999). Classification of solutions of higher order conformally invariant equations, Math. Ann., 313, 207-228. https://doi.org/10.1007/s002080050258

$\mathrm{Xu}, \mathrm{C} .$, \& Tang, T. (2006) Stability analysis of large time-stepping methods for epitaxial growth models, SIAM J. Numer. Anal., 44, 1759 - 1779 https://doi.org/10.1137/050628143

\section{Copyrights}

Copyright for this article is retained by the author(s), with first publication rights granted to the journal.

This is an open-access article distributed under the terms and conditions of the Creative Commons Attribution license (http://creativecommons.org/licenses/by/4.0/). 